\title{
Comunicação
}

[Communication]

\section{Freqüência de helmintos em gatos de Uberlândia, Minas Gerais}

\author{
[Frequency of helminthes parasites in cats of Uberlândia, Minas Gerais]
}

\author{
T.C.D. Mundim, S.D. Oliveira Júnior, D.C. Rodrigues, M.C. Cury*
}

Instituto de Ciências Biomédicas da Universidade Federal de Uberlândia

Av. Pará, 1720, Bloco 4C

38400-902 - Uberlândia, MG

\begin{abstract}
Os gatos são hospedeiros de uma série de parasitos, principalmente de helmintos gastrintestinais. Além da patogenicidade direta ao hospedeiro, eles apresentam risco à saúde humana, pelo fato de parasitarem ambas as espécies. $\mathrm{O}$ gato, por seus hábito $\mathrm{e}$ comportamento peculiares, pode contribuir para a disseminação de determinados parasitos, como, por exemplo, Ancylostoma sp, Toxocara sp. As larvas desses parasitos podem infectar o homem, causando as síndromes conhecidas por larva migrans cutânea e larva migrans visceral, respectivamente. No Brasil, a prevalência dessas parasitoses foi avaliada mediante exame de fezes e contagem direta de parasitos após a necropsia dos hospedeiros. Os resultados indicam que os parasitos de vários gêneros e espécies são amplamente distribuídos pelo país. O objetivo deste estudo foi determinar a freqüência de helmintos parasitos de gatos domésticos no município de Uberlândia, Minas Gerais.
\end{abstract}

Cinqüenta gatos, de ambos os sexos, sem raça definida e de diferentes faixas etárias, sacrificados e doados pelo Centro de Controle de Zoonoses da Prefeitura Municipal de Uberlândia, entre agosto de 2000 e dezembro de 2001, foram necropsiados na Faculdade de Medicina Veterinária da Universidade Federal de Uberlândia.

Recebido para publicação em 31 de julho de 2003. Recebido para publicação, após modificações, 22 de dezembro de 2003.

*Autor para correspondência.

E-mail: cury@umuarama.ufu.br
As cavidades torácica e abdominal foram abertas e os órgãos separados e colocados em sacos plásticos. Os pulmões, seccionados em pequenos fragmentos, acondicionados em placas de Petri com solução salina $0,85 \%$ e incubados à $37^{\circ} \mathrm{C}$ durante 12 horas, foram examinados sob microscópio estereoscópio em aumento de $25 \mathrm{X}$. O fígado, a vesícula biliar e seus ductos foram abertos e lavados com solução salina $0,85 \%$. O trato gastrintestinal foi separado em estômago e intestinos delgado e grosso. Após serem abertos, suas mucosas foram raspadas e lavadas em salina com os respectivos conteúdos, utilizando tamís de abertura de $100 \mathrm{~mm} / \mathrm{mm}$. A contagem e identificação dos helmintos foram feitas segundo Yamaguti (1961) e Travassos (1969).

Quarenta e cinco gatos (90\%) estavam parasitados por um ou mais helmintos e $5(10 \%)$ apresentaram-se negativos. As freqüências das espécies de helmintos encontradas estão na Tab. 1. Platynosomum fastosum e os nematódeos Ancylostoma brazilienses e Physaloptera praeputialis foram os parasitos mais comuns.

A freqüência de infecção por Aelurostrongylus abstrusus é semelhante à observada por Ogassawara et al. (1986) em São Paulo (18,5 \%). Em Belo Horizonte, Costa et al. (1966) verificaram $58,8 \%$ de animais parasitados por esse helminto. Ancylostoma braziliensis e A. caninum apresentaram freqüências semelhantes às observadas por Ogassawara et al. (1986) e Langnegger e Lanzieri (1963/1965) no Rio de Janeiro, porém menores que as apontadas por Costa et al. (1966). Quanto ao Toxocara cati, a freqüência foi superior à encontrada por 
Langnegger e Lanzieri (1963/1965). A presença do Toxocara canis em gatos na cidade de Uberlândia reveste-se de importância pela possibilidade de infecção por larva migrans visceral, especialmente em crianças. Os valores para Dipylidium caninum e Taenia taeniformis foram inferiores aos encontrados por Costa et al. (1966) e Ogassawara et al. (1986) e a freqüência para Physaloptera praeputialis é superior à observada por Ogassawara et al. (1986). Os valores para Platynossomum fastosum seguem a descrição feita por Langnegger e Lanzieri (1963/1965), isto é, 55\% de freqüência. Na literatura não há registro de Spirometra mansonoides, Trichuris campanula e T. vulpis como parasitas de gatos no Estado de Minas Gerais. Das 15 espécies citadas, Dipylidium caninum, Spirometra mansonoides, Toxocara canis, T. cati, Ancylostoma braziliensis e A. caninum podem ser encontradas como parasitas do homem. Portanto, são zoonoses e podem tornar grave o problema de saúde pública.
Tabela 1. Freqüência de helmintos em gatos necropsiados entre agosto/2000 e dezembro de 2001 no município de Uberlândia, Minas Gerais

\begin{tabular}{lcc}
\hline Espécie & $\begin{array}{c}\text { Número de } \\
\text { gatos parasitados }\end{array}$ & $\begin{array}{c}\text { Freqüência } \\
(\%)\end{array}$ \\
\hline Aelurostrongylus abstrusus & 9 & 18 \\
Ancylostoma braziliensis & 19 & 38 \\
Ancylostoma caninum & 7 & 14 \\
Dipylidium caninum & 7 & 14 \\
Physaloptera praeputialis & 17 & 34 \\
Platynossomum fastosum & 20 & 40 \\
Spirometra mansonoides & 2 & 4 \\
Taenia taeniaeformis & 2 & 4 \\
Toxascaris leonina & 2 & 4 \\
Toxocara canis & 5 & 10 \\
Toxocara cati (T. mistax) & 7 & 14 \\
Trichuris campanula & 3 & 6 \\
Trichuris vulpis & 1 & 2 \\
Negativos & 5 & 10 \\
\hline
\end{tabular}

Palavras-chave: gato, helmintos, freqüência, Uberlândia

\begin{abstract}
Necropsies in 50 cats, males and females of different ages were performed from August 2000 to December 2001. Forty five (90\%) of them had parasites. Eleven cats (22\%) had single infection, 34 (75.6\%) multiple infection, and only 5 (10\%) were free of infection. The parasitic frequencies were as follow: Platynosomum fastosum (40\%), Ancylostoma braziliensis (38\%), Physaloptera praeputialis (34\%), Aelurostrongylus abstrusus (18\%), Dipylidium caninum (14\%), Ancylostoma caninum (14\%), Toxocara mistax (14\%), Toxocara canis (10\%), Trichuris campanula (6\%), Toxascaris leonina (4\%), Spirometra mansonoides (4\%), Taenia taeniaeformis (4\%) e Trichuris vulpis $(2 \%)$.
\end{abstract}

Keywords: cat, helminth, frequency, Uberlândia

\section{REFERÊNCIAS BIBLIOGRÁFICAS}

COSTA, H.M.A.; COSTA, J.O.; FREITAS, M.G. Parasitos de Felis domestica em Belo Horizonte, Minas Gerais. Arq. Esc. Vet. UFMG, v.18, p.65-69, 1966.

LANGNEGGER, J.; LANZIERI, P.D. Incidência e intensidade de infestação por helmintos em (Felis catus domesticus) do Rio de Janeiro. Veterinária, v.16-18, p.77-89, 1963/1965.
OGASSAWARA, S.; BENASSI, S.; LARSSON, C.E. et al. Prevalência de infecções helmínticas em gatos na cidade de São Paulo. Rev. Fac. Med. Vet. Zootec. USP, v.23, p.145-149, 1986.

TRAVASSOS, L.; FREITAS, J.F.T.; KONH, A. Trematódeos do Brasil. Mem. Inst. Oswaldo Cruz, v.67, p.11-865, 1969.

YAMAGUTI, S. Systema Helminthum. New York: Interscience Publisher, 1961. 